\title{
Pessoas em situação de rua em Manaus e o direito à saúde: um estudo sobre
} adesão ao tratamento em HIV ${ }^{1}$

\section{Homeless people in Manaus and the right to health: a study on HIV treatment adherence}

DOI: $10.46814 / \operatorname{lajdv3n4-021}$

Recebimento dos originais: 01/05/2021

Aceitação para publicação: 31/06/2021

\section{Rosiane Pinheiro Palheta}

Doutora

Instituição de atuação atual: Fundação Hospital Adriano Jorge

End: Av. Carvalho Leal, n. 1778 - São Francisco. CEP: 69065-001.

E-mail: anypinheiro@ hotmail.com

\section{Raquel Lira de Oliveira Targino \\ Mestre}

Instituição de atuação atual: Secretaria Municipal de Saúde de Manaus

End: Av. Carvalho Leal, n. 1778 - São Francisco. CEP: 69065-001

E-mail: anypinheiro@hotmail.com

\section{Lucélia Regina Pacheco Araújo}

Ensino médio

Instituição de atuação: CEULM/ULBRA- Manaus

End: Av. Carvalho Leal, n. 1778 - São Francisco. CEP: 69065-001

E-mail: anypinheiro@hotmail.com

\section{RESUMO}

No Brasil os estudos sobre o tratamento de saúde com soro-positivos ainda é incipiente, sobretudo no que diz respeito ao processo de adesão ao tratamento que ainda é um fator que tem limitado o sucesso no tratamento antiretroviral. Alguns estudos apontam que a não-adesão é um desafio e uma ameaça para a saúde, tanto individual quanto para a Saúde Pública. Sabe-se ainda que, na perspectiva do paciente, a adesão traz inúmeros benefícios ao tratamento dentre eles destaca-se: a redução do risco de falha virológica, aumenta a sobrevida do paciente, reduz o risco de progressão para AIDS e principalmente a melhora na qualidade de vida. Como está o acompanhamento e a adesão ao tratamento em Manaus e como as pessoas aderem a este serviço, é um dos objetivos da pesquisa aqui apresentada. Os procedimentos metodológicos se basearão nos métodos qualitativos e quantitativos e tratando-se de uma pesquisa descritiva, a metodologia utilizada para coleta de dados, objetivou descrever as características da população soro-positiva atendida nos SAES de Manaus e pela Equipe de Consultório na Rua. Como principais resultados baseados na análise dos dados feita a partir dos dados disponíveis pelo SAE /AHF de 2020 e tomando por base o acompanhamento do Serviço de psicologia e Serviço social, há uma adesão bastante significativa dos pacientes ao tratamento antirretroviral em $85 \%$ dos 903 pacientes em acompanhamento. Em relação aos pacientes em situação de rua atendidos pela Equipe de Consultório na Rua (CNAR), foram acompanhados 07 pacientes nos últimos 6 anos, dentre os quais

\footnotetext{
${ }^{1}$ Projeto de pesquisa realizado para o Programa de Apoio à Iniciação Científica (PAIC), da Fundação Hospital Adriano Jorge (FHAJ), com apoio da Fundação de Aparo à Pesquisa do Amazonas (FAPEAM).
} 
01 foi à óbito devido às complicações com comorbidades como a tuberculose, 01 está em tratamento e os demais não foram mais encontrados pela equipe.

Palavras chave: Soropositivos, Tratamento, Adesão, HIV

\section{RESUMO}

In Brazil, studies on health care treatment with sero-positive individuals is still incipient, especially regarding the treatment adherence process that is still a factor that has limited the success in antiretroviral treatment. Some studies point out that non-adherence is a challenge and a threat to health, both individual and public health. It is also known that, from the patient's perspective, adherence brings several benefits to the treatment, among which we highlight: reduced risk of virologic failure, increased patient survival, reduced risk of progression to AIDS, and especially improved quality of life. How is the monitoring and adherence to treatment in Manaus and how people adhere to this service, is one of the objectives of the research presented here. The methodological procedures will be based on qualitative and quantitative methods and being a descriptive research, the methodology used for data collection, aimed to describe the characteristics of the HIV-positive population assisted in the SAES of Manaus and by the Street Clinic Team. As main results based on data analysis made from the data available by the SAE /AHF of 2020 and based on the monitoring of the Psychology Service and Social Service, there is a very significant adherence of patients to antiretroviral treatment in $85 \%$ of 903 patients in monitoring. Regarding the homeless patients assisted by the Street Clinic Team (CNAR), 07 patients were followed in the last 6 years, among which 01 died due to complications with comorbidities such as tuberculosis, 01 is under treatment and the others were no longer found by the team.

Key words: HIV positive, Treatment, Adherence, HIV

\section{INTRODUÇÃO}

No Brasil os estudos sobre o tratamento de saúde com soro-positivos ainda é incipiente, sobretudo no que diz respeito ao processo de adesão ao tratamento que ainda é um fator que tem limitado o sucesso no tratamento antiretroviral. Alguns estudos apontam que a não adesão é um desafio e uma ameaça tanto para a saúde individual quanto para a Saúde Pública.

Sabe-se ainda que, na perspectiva do paciente, a adesão traz inúmeros benefícios ao tratamento dentre eles destaca-se: a redução do risco de falha virológica, aumenta a sobrevida do paciente, reduz o risco de progressão para AIDS e principalmente a melhora na qualidade de vida. Perno e colaboradores (2002) discutem que uma adesão em níveis ótimos leva a uma replicação mínima de vírus e a uma rara mutação espontânea. Em se tratando de tratamento às pessoas que vivem em situação de rua, essa realidade pode ser ainda mais complexa. Esse trabalho trata do acompanhamento e a adesão ao tratamento em Manaus e como as pessoas aderem a este serviço, sobretudo aquelas pessoas que vivem em situação de rua que é um dos objetivos da pesquisa. 


\section{MATERIAIS E MÉTODOS}

Os procedimentos metodológicos que nortearam a execução da pesquisa incluíram: levantamento bibliográfico, que verificou a existência de produções científicas, trabalhos, investigações e publicações acerca da temática da adesão ao tratamento em HIV no Brasil, dando maior ênfase a produções sobre a região Amazônica, no estado do Amazonas e Manaus.

A pesquisa documental teve como base de dados da vigilância epidemiológica do Ministério da Saúde onde há inúmeras variáveis sobre a incidência de HIV em todo o País e nos Estados da federação.

Tratando-se de uma pesquisa descritiva, a metodologia utilizada para coleta de dados, objetivou descrever as características da população soro-positiva atendida nos SAES de Manaus e pela Equipe de Consultório na Rua.

A pesquisa de campo foi iniciada após aprovação do projeto pelo Comitê de Ética em pesquisa (CAAE 1 14637219.7.0000.0007) que possibilitou a coleta dos dados primários e informações que com os profissionais de saúde envolvidos no cuidado á saúde da população soropositiva.

\section{RESULTADOS E DISCUSSÕES}

A Síndrome de Imunodeficiencia Adquirida (AIDS) é a manifestação avançada do vírus da Imunodeficiencia humana (HIV), mas deve ser compreendida não apenas no contexto de ausência de saúde, mas como um fenômeno social que carrega em si uma carga estigmatizadora e um tabu na qual ainda tem sido um dos fatores que rebatem no sucesso do tratamento do doente ou portador do vírus. Caracteriza-se: por um transtorno da imunidade celular, resultando em maior suscetibilidade a infecções oportunistas e neoplasias1. A transmissão dessa síndrome é predominantemente sexual, embora haja outras formas de exposição ao HIV, tais como a transmissão sanguínea e verticall (COUTINHO et al., 2018, p. 149).

Os dados mais recentes e disponíveis sobre a doença no País são do Boletim Epidemiológico de HIV/AIDS que disponibiliza informações até o primeiro semestre de 2019 pelo Ministério da Saúde. A partir desses dados, é possível identificar algumas características e número de pessoas soro-positivas a nível nacional. Observa-se que desde 2007 a junho de 2019 os homens são significativamente a maior porcentagem de diagnóstico de HIV no País, totalizando desde então 68,95\% de todos os casos, enquanto as mulheres $31,05 \%$ dos diagnósticos registrados. No total são 300.496 casos registrados no Sistema de Informação de Agravos de Notificação (SINAN) desde 2007 sendo 136.902 (45,6\%) somente na região Sudeste, $60.470(20,1 \%)$ na região Sul, 55.090 (18,3\%) na região Nordeste, 26.055 $(8,7 \%)$ na região Norte e $21.979(7,3 \%)$ na região Centro Oeste. 
Gráfico 1 - casos de aids notificados no sinan

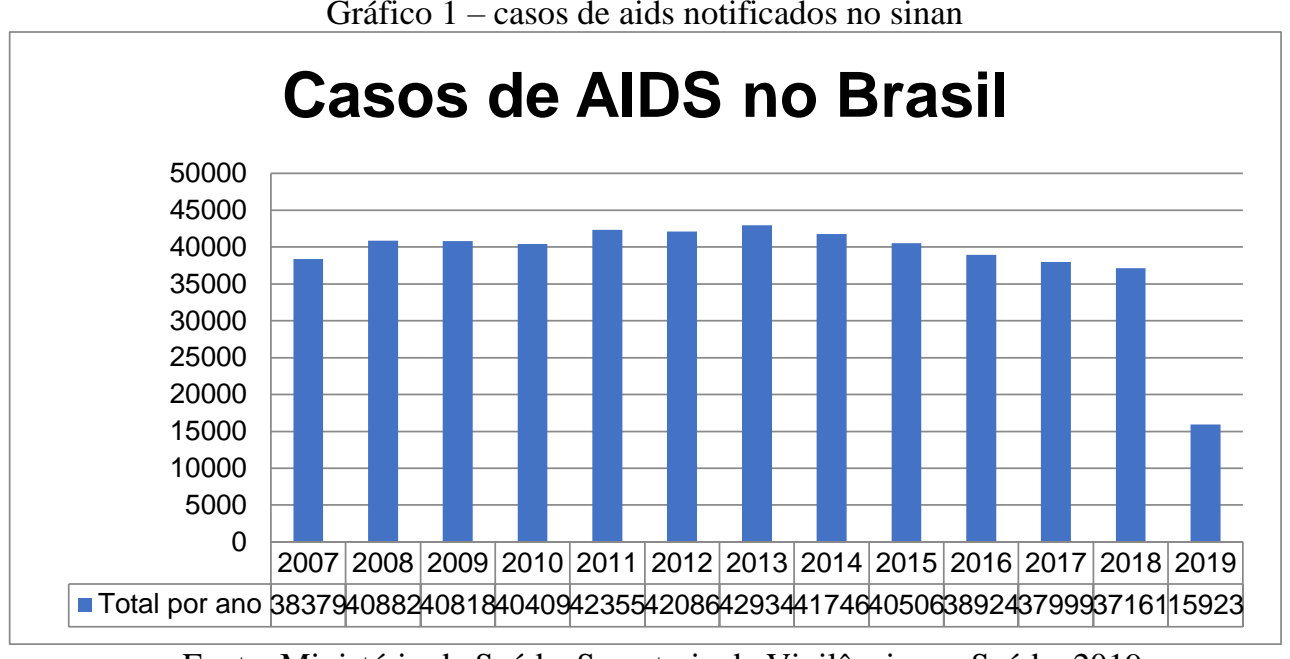

Fonte: Ministério da Saúde, Secretaria de Vigilância em Saúde, 2019

A faixa etária mais infectada pelo vírus do HIV registrada no SINAN em pessoas do sexo masculino é de 25 a 29 anos, correspondendo a 20,5\% e apresentando a tendência a diminuição dessa idade, já em mulheres, apesar da média se manter nessa faixa etária com 15,3\%, a tendência é que cada vez mais essa idade aumente.

Quanto ao Estado do Amazonas, em 2018, o ranking das Unidades Federativas (UF) referente às taxas de detecção de AIDS mostrou que ele figurou, junto com Roraima dentre as maiores taxas, com 29,1 e 40,8 casos por 100.000 habitantes, respectivamente. Além disso, observou-se que outras 11 UF apresentaram taxas superiores à nacional (de 17,8/100.000 habitantes). Minas Gerais foi o estado com a menor taxa: 11,6 casos/100.000 habitantes.

Gráfico 2 - Estado Do Amazonas

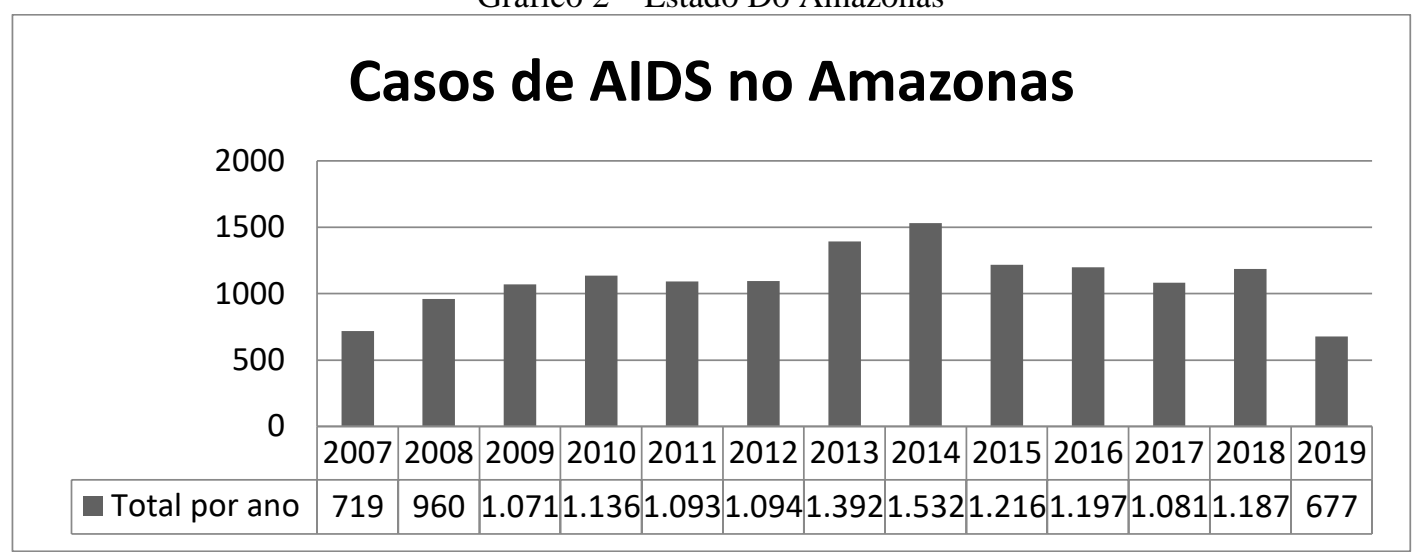

Fonte: Ministério da Saúde, Secretaria de Vigilância em Saúde, 2019

Com relação à infecção em gestantes, o Amazonas aparece entre as Unidades da Federação (UF) com taxa superior à nacional em 2018, juntamente com outras, conforme o descrito a seguir : Rio 
Grande do Sul (9,2 casos/mil nascidos vivos), Santa Catarina (6,1), Roraima (4,6), Rio de Janeiro $(4,1)$, Amazonas (3,5), Pernambuco $(3,4)$, Mato Grosso do Sul $(3,2)$, Amapá $(3,1)$ e Pará $(3,0)$.

GRÁFICO 3 - TAXA DE DETECÇÃO

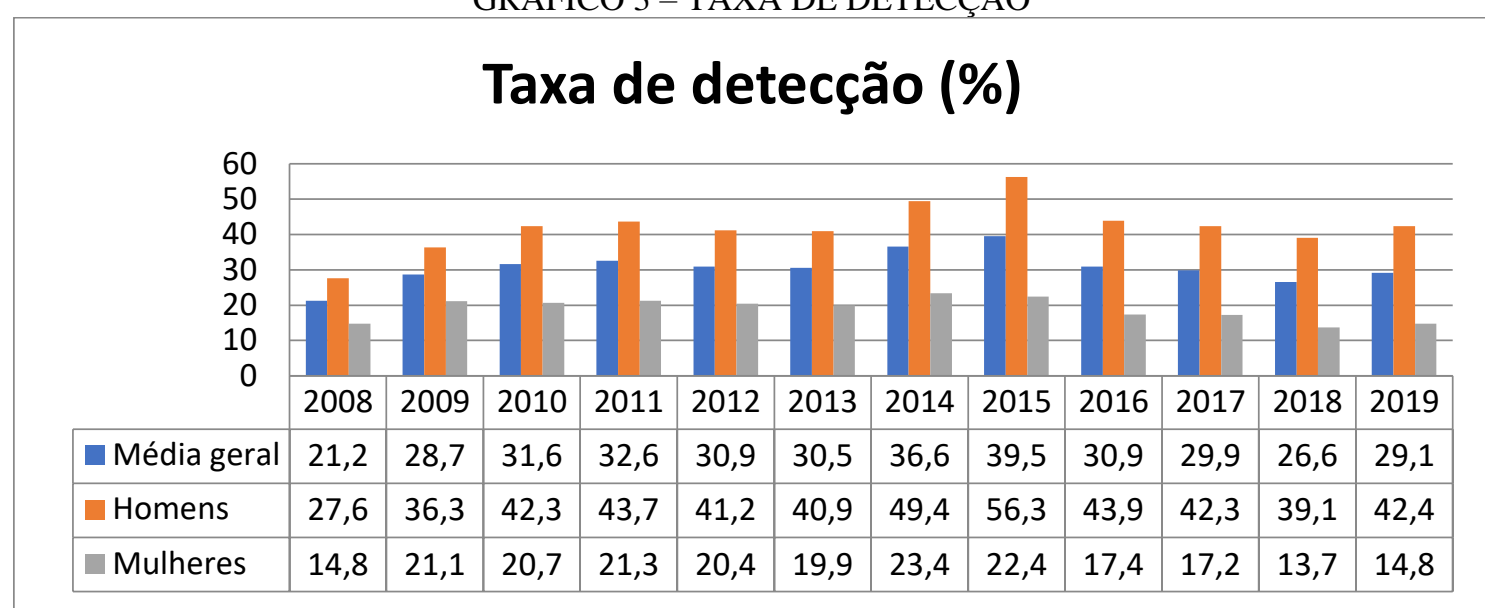

Fonte: Ministério da Saúde, Secretaria de Vigilância em Saúde, 2019

Um dado a ser destacado é a taxa entre homens e mulheres, onde nestas últimas a taxa sempre é inferior a dos homens, o que pode ser explicada por vários fatores associados ou não. Um exemplo disso é a cultura que existe de que mulheres se cuidam mais que os homens ou aderem ao tratamento de forma mais determinante, ou ainda de que os serviços de saúde para o homem estão aquém das políticas para a mulher.

No Programa Conjunto das Nações Unidas sobre HIV/AIDS (UNAIDS), o Relatório Ponto Cego (Blind spot, em inglês) mostra que menos de metade dos homens que vivem com HIV está em tratamento, enquanto que as mulheres têm $60 \%$ do total realizando o tratamento. $\mathrm{O}$ uso do preservativo também é baixo entre os homens dessa população-chave. Quanto à testagem para o HIV, aparenta estar alcançando um grande número de mulheres, sobretudo em serviços de pré-natal, porém, os mesmos pontos de entrada não foram encontrados para os homens.

A cultura machista leva os homens e os meninos a serem influenciados por muitas normas de gênero que podem afetar a saúde sexual e reprodutiva, além de desencorajar o acesso aos serviços de saúde por considerar que as mulheres e crianças são o alvo prioritário das ações de saúde, sobretudo na atenção básica.

Essa $\mathrm{O}$ conceito de masculinidade e os estereótipos associados a ele criam condições que tornam a segurança, sexo, fazer um teste de HIV, acessar e aderir ao tratamento - ou mesmo conversar sobre sexualidade - um desafio para os homens. Hábitos alimentares pouco saudáveis e o uso de álcool, tabaco e drogas exacerbam ainda mais a situação (SIDIBÉ, 2017, p.3).realidade é bem mais presente nas populações de rua, já que a própria condição de estar na rua é um agravante para a busca dos 
serviços e o acesso ao tratamento, bem como de muitos casos positivos dentre essa população que não são identificados e nem tratados na rede de saúde.

O relatório ponto cego mostra ainda uma questão que é cultural no Brasil, onde os homens buscam tratamento de saúde e frequentam as unidades de saúde com menos frequência do que as mulheres, assim como realizam menos exames e são diagnosticados mais tardiamente que as mulheres em condições que já representam risco para a vida.

Neste trabalho foram considerados não aderentes os pacientes que estão há pelo menos seis meses sem ir à consulta ou quando está, há pelo menos, três meses sem retirar a medicação na farmácia da unidade de saúde de referência.

$\mathrm{Na}$ análise dos dados feita a partir dos dados disponíveis pelo SAE /AHF de 2020 e tomando por base o acompanhamento do Serviço de psicologia e Serviço social, há uma adesão bastante significativa dos pacientes ao tratamento antirretroviral em 85\% dos 903 pacientes em acompanhamento.

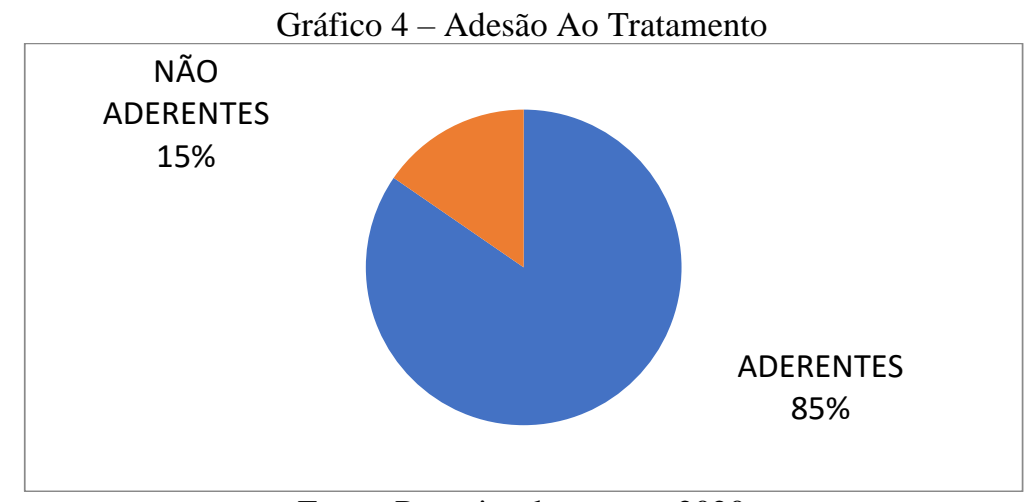

Fonte: Pesquisa de campo, 2020

Em relação aos pacientes em situação de rua atendidos pela Equipe de Consultório na Rua (CnaR), foram acompanhados 7 pacientes nos últimos 6 anos, dentre os quais 01 foi à óbito devido ás complicações com comorbidades como a tuberculose, 01 está em tratamento e os demais não foram mais encontrados pela equipe.

O momento de recebimento do diagnóstico de HIV é crítico em razão da gravidade da doença e do comprometimento do prognóstico, e pode trazer desdobramentos importantes para a vida do sujeito, para seu tratamento e consequentemente para a própria adesão ao tratamento. $\mathrm{O}$ cuidado de comunicar o diagnóstico e a interação com o profissional de saúde nesse momento é decisivo, visto que a emergência de sentimentos de desamparo pode propiciar que os pacientes se apropriem do que é dito pelos profissionais de saúde para definir projetos de vida (COUTINHO, 2018, p.154). 
Um estudo realizado pela equipe $\mathrm{CnaR}$ mostrou que o recebimento do diagnóstico teve efeito devastador na vida de algumas pessoas em situação de rua, levando à revolta por culpar seus parceiros ou parceiras até á tentativa de suicídio (PALHETA et al., 2019).

Um estudo feito por Bonolo et al. (2007) aponta que, entre as estratégias de intervenção para uma adesão maior dos pacientes, o próprio serviço de saúde e a relação estabelecida entre profissionais e pacientes é um ponto crítico e motivador de incremento e manutenção da adesão. Deve-se buscar a participação ativa do paciente no desenvolvimento de habilidades para lidar com um regime terapêutico complexo e de difícil adaptação à vida de cada sujeito em tratamento para o HIV ou AIDS (BONOLO et al., 2007, p. 262).

A doença guarda em si uma estigmatização. Após descobrir a infecção, o doente passa a viver um sofrimento sob vários pontos de vista. De um lado, o sofrimento físico de uma doença que, mesmo com os avanços da ciência, ainda não tem cura, enquanto do outro, o sofrimento psíquico gerado pelo fato de ainda guardar a ideia de que o diagnóstico é uma sentença de morte. Do outro lado tem o sofrimento social causado pelo olhar excludente, pela intolerância, pelo medo e preconceito para com as pessoas que convivem com o vírus.

Segundo Coutinho, a depressão é o transtorno psiquiátrico prevalente em indivíduos infectados pelo HIV12. Ainda que estudos considerem uma variação da prevalência da depressão em Pessoas Vivendo com HIV/AIDS (PVHA) entre 0 e 42\%, há consenso de que ela incide duas vezes mais nessas pessoas do que na população em geral, podendo-se observar uma porcentagem de onze a $30 \%$ nos portadores da infecção (COUTINHO et al., 2018, p. 150).

Na população de rua o sofrimento é clássico e muitas vezes é o motivo que levou as pessoas à situação de rua. O sofrimento psíquico é muito presente nas pessoas vivendo com HIV e muitas vezes ele não é claro levando muitos à depressão que, na grande maioria dos casos não é tratada devido a certo receio em ainda se falar da doença.

No que se refere à teoria das representações sociais, esta tem sido muito utilizada para explicar a problemática psicossocial da AIDS, uma vez que permite compreender o sentido atribuído para um fenômeno como o adoecimento, orientando as condutas dos sujeitos a partir das representações que construíram sobre a doença. Isso acontece porque essas representações sociais se inscrevem em uma experiência humana que apresenta uma série de significados. Ou seja, por meio do estudo das representações torna-se possível compreender a visão de mundo que a pessoa soropositiva constrói a partir da sua inserção em grupos sociais, além de melhor entender a tomada de posição e os comportamentos adotados diante do processo de adesão, o que viabiliza a formação de um elo consistente entre profissional e usuário. 
Dessa forma, a AIDS é percebida como uma doença que traz, além do preconceito e discriminação porque existe um receio e desconhecimento sobre ela, uma série de incertezas e dúvidas em relação ao tratamento e ao futuro. Neste contexto, nota-se a inserção da terapia antirretroviral a partir de 1996, que trouxe consigo uma esperança de prolongar a vida, dando à doença um caráter evolutivo crônico, potencialmente controlado. No entanto, a adesão ao tratamento destaca-se entre os maiores desafios da atenção às pessoas vivendo com HIV/AIDS, uma vez que requer mudanças comportamentais, dietéticas, o uso de diversos medicamentos por toda a vida, além da necessidade, por parte dos serviços de saúde, de novos arranjos e oferta de atividades específicas visando promover e assegurar a adesão.

Almeida e Labronici (2006) afirmam que parece claro a despeito dos avanços médicos e científicos em relação à doença, dentre eles o advento dos antiretrovirais que melhoraram a qualidade de vida das pessoas, afastando-as da grande letalidade da doença, ainda prevalece a constatação de que a epidemia é soberana e que o convívio com o HIV e a AIDS ainda continua cobrando um alto custo aos doentes com um enorme sofrimento humano resultante do estigma, preconceito e discriminação.

A história do tratamento antiretroviral tem seu início no uso de monoterapia com idovudina (AZT), de 1994 a 1996, consolidando-se com a terapia dupla como padrão terapêutico. A partir de 1996, com a terapia tríplice, de introdução dos inibidores da protease os novos regimes terapêuticos têm demonstrado a capacidade de diminuir ou mesmo de tornar indetectável a carga viral do HIV e reduzir a morbidade e mortalidade relacionada ao doente, nesse sentido a adesão é potencialmente capaz de reduzir o risco da transmissão do HIV e de resistência aos medicamentos antiretrovirais. " $A$ transmissão de cepas virais resistentes é um problema em expansão, fortemente relacionado com a não-adesão ao tratamento” (BONOLO et al., 2007, p. 262).

Um grande problema em relação ao tratamento de HIV/AIDS é a adesão ao tratamento, que é um processo colaborativo que facilita a aceitação e a integração de determinado regime terapêutico no cotidiano das pessoas em tratamento, pressupondo sua participação nas decisões sobre o mesmo. Consiste em um processo de negociação no qual se busca a adequação aos hábitos e necessidades individuais e que visa fortalecer a autonomia para o autocuidado, transcendendo, assim, a simples ingestão de medicamentos.

Melchior et al. (2007) em um estudo sobre os desafios da adesão ao tratamento em HIV no Brasil, relatam que uma das dificuldades no tratamento referidas pelos participantes da pesquisa é com relação ao uso da medicação, no que se relaciona aos efeitos colaterais, tais como náusea, vômito, dores de cabeça e diarréia. Bem como dificuldades diretamente relacionadas ao uso de medicação como dificuldade em dissolvê-la ou engoli-la, e intolerância ao cheiro ou gosto. 


\section{CONSIDERAÇÕES FINAIS}

Neste trabalho a adesão ao tratamento mostrou ter níveis elevados $(85 \%)$ com relação aos pacientes atendidos na policlínica Antônio Reis, entretanto é perceptível que ainda existe um quantitativo expressivo de pacientes que abandonam o tratamento naquela unidade de saúde de referência. Quando se olha para a população de rua essa realidade é ainda mais crítica.

Alguns fatores podem estar envolvidos nessa situação, primeiramente pela instabilidade de vida e de rotina em que vivem. Não têm lugar fixo para estar, são nômades na cidade, o que dificulta um acompanhamento eficaz da equipe itinerante e, por outro lado, a unidade de referência não tem muito controle sobre essa população, já que não tem endereço e nem um apoio familiar de contato.

Os serviços de saúde estão buscando estratégias para maior adesão e o CnaR pode ser considerado um serviço e uma estratégia interessante como via de acesso das populações excluídas aos serviços essenciais de saúde, porém, ainda há um longo caminho a ser percorrido para minimizar os obstáculos inerentes à complexidade do fenômeno que a rua engendra pois esta é reflexo e imagem da complexa teia de questões sociais existentes em nosso país. 


\section{REFERÊNCIAS}

PERNO, C. F., CECCHERINI-SILBERSTEIN, F., DE LUCA, A., COZZI-LEPRI, A., GORI, C., CINGOLANI, A. et al. Virologic correlates of adherence to antiretroviral medications and therapeutic failure. Journal of Acquired Immune Deficiency Syndromes. 2002;31:S118-S122.

BRASIL. Ministério da Saúde. Programa Nacional de IST e AIDS. Boletim epidemiológico de HIV e Aids. Brasília, Secretaria de Vigilância em Saúde, 2019.

ALMEIDA, M. R. C. B., LABRONICI, L. M. A trajetória silenciosa de pessoas portadoras do HIV contada pela história oral. Revista Ciência e Saúde Coletiva, 2006.

COUTINHO, et al. Tratamento antirretroviral: adesão e a influência da depressão em usuários com HIV/AIDS atendidos na atenção primária. Saúde em debate, 2018.

MELCHIOR, Regina, et al. Challenges of treatment adherence by people living with HIV/AIDS in Brazil. Revista Saúde Pública, 2007.

BRASIL. Programa Conjunto das Nações Unidas sobre HIV/AIDS (UNAIDS). Ponto Cego. 2017.

BEZERRA, JR, B. Os desafios da reforma psiquiátrica no Brasil. Revista de Saúde Coletiva, v. 17, n. 22, p. 243-250, 2007.

BONOLO, P. F., et al. Adesão à terapia anti-retroviral (HIV/aids): fatores associados e medidas da adesão. Epidemiologia Serviço em Saúde, Brasília, 2007.

PALHETA, R. P., et al. (SOBRE)VIVÊNCIAS NAS RUAS DE MANAUS: histórias, condições de vida e políticas de saúde. Editora CRV, Manaus, 2019.

Sidibé, Michel. HIV: preconceito leva homens a se descuidar do tratamento. In. Vida e ação, saúde, bem estar e atitude sustentável. 2017. Disponível em https://www.vidaeacao.com.br/hivpreconceito-leva-homens-a-descuidar-da-saude/. 